\title{
El fitoplancton de Lagunas de Ruidera (Parque Natural, Ciudad Real, España)
}

\author{
Sara Bort ${ }^{1}$, Carmen Rojo ${ }^{1}$, María Antonia Rodrigo ${ }^{1}$ y Nora Maidana ${ }^{2}$ \\ ${ }^{1}$ Instituto Cavanilles de Biodiversidad y Biología Evolutiva, Universidad de Valencia. Apartado Oficial 2085. \\ 46071 Valencia, España. saradelarco@hotmail.com \\ ${ }^{2}$ Dpto. de Ciencias Biológicas, Universidad de Buenos Aires. C1428EHA, Buenos Aires, Argentina.
}

\section{RESUMEN}

El Parque Natural Lagunas de Ruidera es una zona húmeda catalogada como de importancia nacional situada en el valle del Alto Guadiana, en Ciudad Real. Está constituido por 15 lagunas separadas por barreras travertínicas, acabando en el embalse de Peñarroya. En este estudio se presentan los primeros datos conocidos sobre la biodiversidad, abundancia, biovolumen y dinámica del fitoplancton (incluido el picoplancton autotrófico) de este sistema. Las muestras se tomaron en mayo y septiembre de 2000 y en marzo, junio, septiembre y diciembre de 2001 en el embalse y en cada laguna, excepto en aquellas que sufrieron periodos secos. Todas las muestras fueron tomadas del epilimnion.

El estudio taxonómico del fitoplancton permitió evidenciar la presencia de 215 especies pertenecientes a los grandes grupos de algas: 9 taxones a Cyanophyta, 9 a Euglenophyta, 6 a Cryptophyceae, 7 a Dinophyceae, 10 a Chrysophyceae, 58 a Chlorophyta, 103 Bacillariophyceae, 12 a Desmidiaceae, y un grupo de pequeños flagelados considerados como una única población. Las especies más comunes fueron: Cyclotella ocellata, Rhodomonas minuta, Cryptomonas erosa, Cyclotella kuetzingiana y Peridinium umbonatum. Las lagunas presentaron una alta riqueza específica propia de sitios aun no muy degradados. Los grupos funcionales de las algas dominantes (Reynolds, 1997) fueron propios de sistemas eutróficos: Y (Cryptomonas; pequeños lagos eutróficos), L (Peridinium umbonatum y Ceratium; lagos meso-eutróficos estratificados), C (Asterionella formosa y Aulacoseira; lagos eutróficos mezclados) y E (Dinobryon; lagos oligo-mesotróficos).

La dinámica temporal de la abundancia y composición del fitoplancton fue diferente en cada laguna. El biovolumen calculado del fitoplancton se encontró entre 0.2 y $7.2 \mathrm{~mm}^{3} / 1$, y el del picoplancton autotrófico entre 0.12 y $0.2 \mathrm{~mm}^{3} / 1$. Tanto la biomasa algal como la concentración de clorofila $a$ resultaron menores de lo esperado para la elevada concentración de nutrientes medida en las lagunas. Sin embargo, el aporte relativo del picoplancton y la composición algal indican un alarmante proceso de eutrofización.

Palabras clave: Lago kárstico, picoplancton autotrófico, eutrofización, grupos funcionales, dinámica del fitoplancton.

\begin{abstract}
The Lagunas de Ruidera Natural Park is a humid zone classified as of national importance, located in the alto de Guadiana valley, Ciudad Real. The Park is composed of 15 lagoons separated by travertine barriers, which end in the Peñarroya reservoir. This work shows the first published data on biodiversity, density, biomass and dynamics of phytoplankton (including autotrophic picoplankton) of this system.

Samples were collected in May and September 2000 and in March, June, September and December 2001 in the reservoir and in each lagoon (except in those which suffered a dry period). All samples were collected from the epilimnion.

The taxonomic study revealed the presence of 215 species: 9 taxa of Cyanophyta, 9 of Euglenophyta, 6 of Cryptophyceae, 7 of Dinophyceae, 10 of Chrysophyceae, 58 of Chlorophyta, 103 of Bacillariophyceae, 12 of Desmidiaceae, and a group of small fragellates considered to be a single population. The most common species were: Cyclotella ocellata, Rhodomonas minuta, Cryptomonas erosa, Cyclotella kuetzingiana and Peridinium umbonatum. The lakes had a high species richness associated with non contaminated places. The functional groups of dominant algae (Reynolds, 1997) were indicative of eutrophic systems: Y (Cryptomonas; small eutrophic lakes), L (Peridinium umbonatum and Ceratium; stratified meso-eutrophic lakes), $C$ (Asterionella formosa and Aulacoseira; mixed eutrophic lakes) and E (Dinobryon; oligo-mesotrophic lakes). The temporal dynamics of abundance and composition of phytoplankton was different in each lagoon. The estimated phytoplankton biomass range was between 0.2 and $7.2 \mathrm{~mm}^{3} / \mathrm{l}$, and that of the autotrophic picoplankton was between 0.12 and $0.2 \mathrm{~mm}^{3} / \mathrm{l}$. Both algae biomass and chlorophyll a concentration were lower than expected in relation to the high nutrient concentrations in the lagoons. However, the relative contribution of picoplankton and algal composition, indicate an alarming eutrophication process.
\end{abstract}

Keywords: Karstic lake, autotrophic picoplancton, eutrophication, functional groups, phytoplancton dynamic. 


\section{INTRODUCCIÓN}

Los sistemas lacustres pueden ser ecosistemas de elevada riqueza específica, de gran relevancia ecológica y social. En España, el Parque Natural de las lagunas de Ruidera es una zona húmeda catalogada de importancia nacional (Aragón, 2001) situada en el valle del Alto Guadiana, en los límites de Ciudad Real y Albacete. Está constituido por 15 lagunas separadas por barreras travertínicas, acabando en el embalse de Peñarroya. Su principal aporte de agua es subterráneo (Montero, 2000).

En este estudio se hace una aproximación al conocimiento de la biodiversidad, abundancia y biovolumen del fitoplancton (incluido el picoplancton autotrófico) de este sistema lagunar. La importancia de este trabajo se debe a la inexistencia de estudios recientes sobre el plancton en estas lagunas, que aún presentan un buen estado de conservación, y al hecho de integrar un proyecto multidisciplinar que, por ejemplo, ha permitido conocer su estado de contaminación (ÁlvarezCobelas et al., 2002; Álvarez- Cobelas, 2002).

Otros estudios recientes en Ruidera han abordado sus características físicas (Pedley et al., 1996; Andrews et al., 2000) y las poblaciones piscícolas (Elvira et al., 1996; Perdices et al., 2000). Estos y nuevos estudios permitirán tener un mayor conocimiento de la dinámica de este ecosistema y del impacto que la eutrofización pueda provocar, y por tanto ayudarán a tomar medidas para su uso sostenible y su conservación.

Durante los últimos años se han realizado en España estudios en otros sistemas lacustres cársticos, como es el caso de la laguna de la Cruz, donde se han realizado trabajos sobre distribución vertical y variación estacional del plancton (Rojo \& Miracle, 1987; Dasí \& Miracle, 1991; Armengol \& Miracle, 2000) y sobre bacterias (Rodrigo et al., 2000). También en Pirineos, Cuenca y Girona (Miracle et al., 1992), y menos recientemente en otras lagunas manchegas, incluidas las de Ruidera (Armengol et al., 1975). A escala internacional, se han realizado trabajos de investigación limnológica en sistemas acuáticos cársticos muy similares a
Ruidera, como es el caso los ríos Sava y Kupa (Croacia), donde han sido estudiados los ciliados (Primc-Habdija et al., 1996 y 2001) y los macroinvertebrados (Habdija, 1997; Matonickin et al., 2001), respectivamente.

\section{MATERIAL Y METODOS}

\section{Sitio de estudio}

El Parque Natural de las lagunas de Ruidera es un paisaje cárstico formado por un rosario de 15 lagunas interconectadas, cerradas por barreras tobáceas que represan sus aguas (excepto las lagunas Colgada y Rey, que fueron unidas artificialmente) y que acaba en el embalse de Peñarroya (Fig. 1). Algunas presentan márgenes acantilados; otras, orillas suaves con carrizales y espadañales (García et al., 1997). El agua fluye en sentido sureste a noroeste, y proviene de manantiales laterales y aportes subterráneos procedentes del acuífero del Campo de Montiel, y de los aportes superficiales del Alto Guadiana (Grande, 1997). Las precipitaciones son escasas e irregulares, siendo las estaciones más lluviosas invierno y primavera, dándose sequía en verano (típico de climas templados cálidos). La media anual de precipitación es de $466 \mathrm{~mm}$. La evapotranspiración potencial anual es de $7751 / \mathrm{m}^{2}$ (Serrano \& García, 1997).

La laguna de mayor superficie es Colgada (100 Ha); las que menor superficie presentan

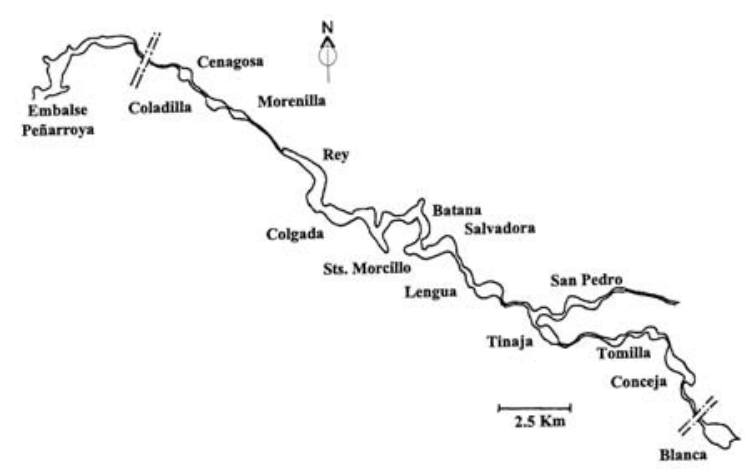

Figura 1. Mapa del Parque Natural de las lagunas de Ruidera. Map of Natural Park of Lagunas de Ruidera. 

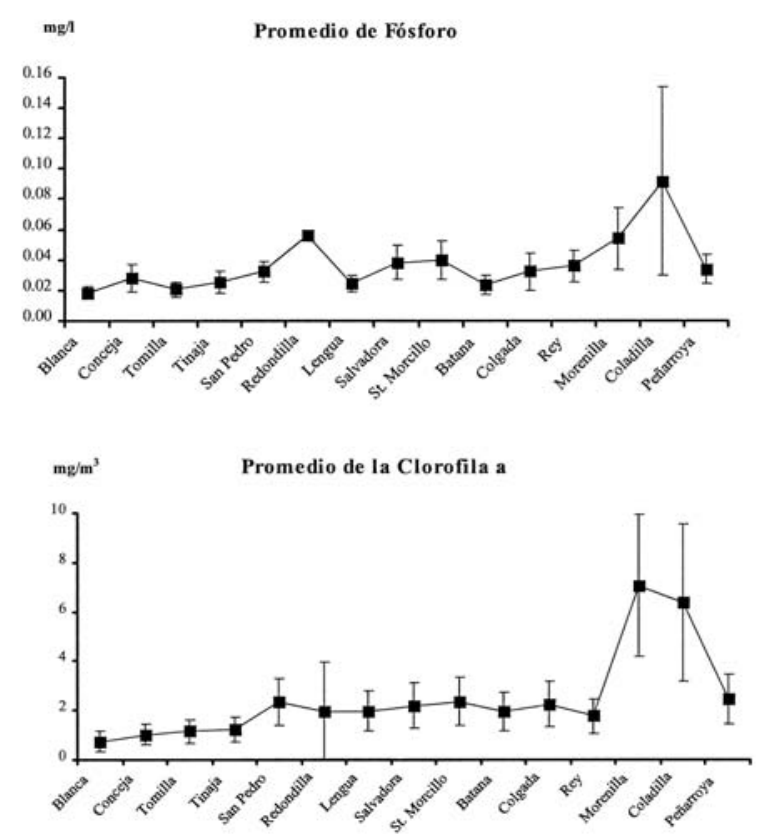

Figura 2. Variabilidad de las concentraciones de fósforo total y clorofila $a$ en el Parque Natural de las lagunas de Ruidera desde la primavera de 2000 al invierno de 2001. Las barras indican la amplitud de la desviación típica. Variability of total phosphorus and chlorophyll a concentrations in the Natural Park of Lagunas de Ruidera from spring 2000 to winter 2001. Bars show the amplitude of typical deviation.

(menos de $12 \mathrm{Ha}$ ) son Tomilla, Tinaja y Redondilla (lagunas altas), Salvadora, Santo Morcillo y Batana (lagunas medias), y Morenilla y Coladilla (lagunas bajas). El resto presentan superficies entre las $38 \mathrm{Ha}$ de Rey y las $22 \mathrm{Ha}$ de Blanca. En cuanto a sus profundidades máximas, en Tinaja, San Pedro, Colgada y Rey se alcanzan los $19 \mathrm{~m}$ de media, siendo las más profundas; Batana, Morenilla y Coladilla son las menos profundas $(8 \mathrm{~m})$; en el resto las profundidades varían de 10 a $14 \mathrm{~m}$. Redondilla es la más somera, secándose durante las épocas de sequía. El embalse de Peñarroya es el más extenso y profundo, con 400 Ha y $30 \mathrm{~m}$ de profundidad.

El estado trófico de las lagunas y el embalse (Fig. 2), caracterizado por las concentraciones de fósforo total y clorofila a (ÁlvarezCobelas et al., 2002 y com.pers.) varía durante el año de la oligotrofia a la eutrofia según la clasificación de la OCDE (1982).

\section{Muestreos y análisis de las muestras}

Las muestras se tomaron en mayo y septiembre de 2000 y en marzo, junio, septiembre y diciembre de 2001 en cada laguna (en el epilimnion), excepto en las lagunas Blanca, Redondilla y Cenagosa que presentaron periodos secos. Las muestras se recogieron en botes de $250 \mathrm{ml}$, que inmediatamente fueron fijadas con lugol; las muestras de picoplancton autotrófico (PPA) se fijaron con formol al 1-2\%. Al mismo tiempo se tomaron muestras y datos para el estudio de los parámetros físico-químicos (Álvarez- Cobelas et al., 2002; Riolobos et al., 2002).

Para el estudio del fitoplancton, las muestras de agua se sedimentaron en cámaras Utermöhl, para posteriormente hacer la identificación y recuento de las poblaciones en un microscopio invertido Olympus CK2 a 400x y 1000x. Se contaron al menos 500 individuos del alga más abundante $(9 \%$ de error según Lund et al., 1958) y se midieron 10 individuos de cada población para determinar sus tamaños. El volumen celular se calculó empleando las formas geométricas de Rott (1981). Un estudio más detallado de la composición taxonómica de las diatomeas se hizo siguiendo las recomendaciones de Hasle \& Fryxell (1970). Las obras de consulta empleadas para la determinación taxonómica fueron: las series Das Phytoplankton des Süßwasserflora (Schweizerbart'sche Verlagsbuchhandlung, Stuttgart) y Süßwasserflora von Mitteleuropa (Gustav Fischer Verlag, Stuttgart); las monografías de Tell \& García (1986), Comas (1996) y Komárek \& Jankovská (2001), y varios artículos: Anton \& Duthie (1981), Hindák (1987), Comas \& Krienitz (1997) y Rosaluz \& Comas (2000).

El PPA fue recontado por la técnica de autofluorescencia según MacIsaac \& Stockner (1993). El biovolumen se calculó usando la misma metodología que para el del fitoplancton.

\section{Análisis estadísticos de los datos}

Se realizaron análisis de regresión y ANOVA de un factor, ambos análisis utilizando el paquete informático SPSS-10, y las recomendaciones de Sokal \& Rohlf (1986): 
La regresión se llevó a cabo para estudiar la relación entre el biovolumen total de fitoplancton, y las concentraciones de clorofila $a$ y fósforo total. Los datos fueron normalizados logarítmicamente.

El ANOVA de un factor se realizó para determinar si había diferencias entre los biovolúme- nes primaverales fitoplanctónicos en las lagunas con mayor o menor cobertura de carófitos (de desarrollo en primavera). Para ello se consideraron dos grupos: lagunas que presentaban entre 0 y $5 \mathrm{~m}^{2}$ de cobertura vegetal/ $\mathrm{m}^{2}$ de superficie de laguna; y aquellas con mas de $5 \mathrm{~m}^{2}$.

Tabla 1. Lista de especies del fitoplancton de las lagunas de Ruidera y el embalse de Peñarroya (2000-2001). Densidades en 10-3 ind./1. + significa densidad inferior a 0.5 ind./1. Se incluye un listado con las especies de diatomeas identificadas en un estudio taxonómico exhaustivo. List of phytoplankton species of Lagunas de Ruidera and the Peñarroya reservoir (2000-2001). Densities in 10-3 ind./l. + means density less than $0.5 \mathrm{ind} . / \mathrm{l}$. A list of diatom species identified in an exhaustive taxonomic study is included.

\section{\%OCURR MIN DENS MAX DENS}

\section{Cianoficeas}

Aphanizomenon flos-aquae (Linn.) Ralfs ex Bornet \& Flahault Limnothrix redekei (Van Goor) Meffert

Limnothrix sp.

Merismopedia glauca (Ehrenberg) Kützing

Microcystis botrys Teiling

Microcystis sp.

Planktothrix agardhii (Gomont) Anagnostidis \& Komárek

Pseudanabaena limnetica (Lemm.) Komarek

Synechocystis aquatilis Sauvageau

\section{Euglenofitas}

Astasia sp.

Euglena acus Ehrenberg

Euglena gracilis Klebs

Euglena oxyuris Schmarda

Lepocinclis ovum var. globula (Perty) Lemm.

Trachelomonas hispida (Perty) Stein emend. Defl.

Trachelomonas intermedia Dang.

Trachelomonas tuberculata Middelh.

Trachelomonas volvocinopsis Swir.

\section{Criptofíceas}

Chroomonas sp.

Cryptomonas erosa Ehrenberg

Cryptomonas marsonii Skuja

Cryptomonas phaseolus Skuja

Cryptomonas rostratiformis Skuja

Rhodomonas minuta Skuja

9

6

31

2

1

14

16

22

20

\section{2}

4

4

1

1

1

\section{Dinofíceas}

Ceratium hirundinella (O. F. Müller) Dujardin

Gymnodinium helveticum Penard

Gymnodinium uberrimum (Allman) Kofoid \& Swezy

Gymnodinium wawrikae Schiller

Katodinium fungiforme (Anisimova) Loeblich III

Peridinium umbonatum Stein

Peridinium willei Huitfeld-Kaas



13

171

67

68

3

494

40

53

7117

4

1

93

13

14

$+$

1

273

1

83

568

747

681

45

12630

$+\quad 40$

232

$+25$

$+\quad 50$

$1 \quad 40$
+

$+525$

$+\quad 50$ 
Tabla 1. Continuación. Continuation.

\section{\%OCURR MIN DENS MAX DENS}

\section{Crisofíceas}

Bitrichia ochridana (Fott) Bourrelly

Bitrichia ollula (Fott) Bourrelly

Chrysococcus ornatus Pascher

Chrysolykos planctonicus Mack

Dinobryon crenulatum W. et G. S. West

Dinobryon divergens Imhof

Dinobryon sertularia Ehrenberg

Mallomonas sp.

Ochromonas viridis Böcher

Ochromonas sp.

2
10
23
26
58
6
19

Ankistrodesmus gracilis (Reinsch) Kors.

Ankistrodesmus spiralis (Turn.) Lemm.

Carteria sp.

Chlamydomonas cf. subfusiformis Gerloff

Chlamydomonas ehrenbergii Goroschankin

Chlamydomonas sp.

Chloromonas sp.

Closteriopsis acicularis (G.M. Smith) Belcher \& Swale

Closteriopsis longissima (Lemm.) Lemm.

Coelastrum astroideum De-Not.

Coelastrum indicum Turner

Coelastrum microporum Näg. in A. Br.

Coelastrum printzii Rayss

Coelastrum reticulatum (Dang.) Senn

Crucigenia tetrapedia (Kirchn.) W. \& G. S. West

Crucigeniella rectangularis (Näg.) Kom.

Dictyosphaerium pulchellum Wood

Dictyosphaerium $\mathrm{sp.}$

Elakatothrix genevensis (Reverd.) Hind.

Kirchneriella contorta var. elegans (Playf.) Komarek

Lagerheimia genevensis (Chod.) Chod.

Lagerheimia subsalsa Lemm.

Monoraphidium arcuatum (Kors.) Hind.

Monoraphidium convolutum (Corda) Kom.-Legn.

Monoraphidium irregulare (G. M. Smith) Kom.-Legn.

Monoraphidium komarkovae Nygaard

Monoraphidium minutum (Nägeli) Komárkova-Legnerová

Monoraphidium subclavatum Nyg.

Monoraphidium tortile (W. \& G.S. West) Kom.-Legn.

Oocystis cf. lacustris Chod.

Oocystis sp.

Pediastrum boryanum (Turp.) Menegh.

Pediastrum boryanum var. longicorne Reinsch

Planktonema lacustris

11

2

10

7

23

26

58

6

2

19

1

2

1

6

4

4

12

4

9

5

1

21

1

4

21

10

22

7

31

2

21

6

1

16

2

11

21

1

11

43

4

11

1

28

Scenedesmus aculeolatus Reinsch

Scenedesmus acuminatus (Lager.) Chodat var. acuminatus Lagerh.

Scenedesmus acuminatus v. minor G. M. Smith

Scenedesmus acutiformis Schröd.

Scenedesmus acutus Meyen$$
2
$$

2 53

8

40

13

114

1625

10636

20

167

83

7

8

1

53

40

11

23

178

39

114

21

382

6

431

254

225

3766

647

982

50

267

13

2

157

3

582

546

26

47

1302

54

10

3

13554 
Tabla 1. Continuación. Continuation.

MAX DENS

Scenedesmus bicaudatus Dedus.

Scenedesmus calyptratus Comas

Scenedesmus disciformis (Chod.) Fott \& Kom.

Scenedesmus ecornis (Ehrenberg) Chodat

Scenedesmus granulatus W. \& G.S.West

Scenedesmus linearis Komarek

Scenedesmus obliquus (Turpin) Kützing

Scenedesmus ovalternus Chod.

Scenedesmus quadricauda (Turpin) Brébisson sensu Chodat

Scenedesmus semipulcher Hortob.

Scenedesmus smithii Teil.

Scenedesmus spinosus Chodat

Schroederia robusta Kors.

Tetraedron caudatum (Corda) Hansg.

Tetraedron minimum (A. Br.) Hansg.

Tetraedron triangulare Kors.

Tetraselmis cordiformis (Carter) Stein

Tetraselmis sp.

Tetrastum triangulare (Chod.) Kom.

Diatomeas

Asterionella formosa Hassall

Aulacoseira sp.

Cyclotella kuetzingiana Thwaites

Cyclotella ocellata Pantocsek

Navicula capitatoradiata Germain

Navicula cryptocephala Kützing

Navicula placentula (Ehrenberg) Grunow

Navicula veneta Kützing

Navicula viridis (Nitzsch) Ehrenberg

Navicula vulpina Kützing

Nitzschia gracilis Hantzch

Nitzschia palea (Kützing) W. Smith

Nitzschia sigmoidea (Nitzsch) W. Smith

Synedra ulna (Nitzsch) Ehrenberg

\section{Desmidiaceas}

Cosmarium bioculatum (Bréb.) ex Ralfs

Cosmarium formosulum Hoff.

Cosmarium humile (Gay) Nordst.

Cosmarium laeve Rabenh.

Cosmarium meneghinii Bréb.

Cosmarium pusillum Hilse

Cosmarium quadratulum (Gay) De Toni

Cosmarium reniforme (Ralfs) Arch.

Cosmarium venustum (Bréb.) Archer

Cosmarium vexatum $\mathrm{W}$. West.

Staurastrum planctonicum Teiling

Otras algas no identificadas

Flagelados pequeños
$\%$ OCURR

MIN DENS

\section{2}

\section{MIN DENS}

45

1

5

17

1

16

1

2

21

2

2

20

2

6

10
612
13
1
9
2
6
3
3
5
8
6
5
2
1
1
1
1
2

612

160

1883

9

471

6

46

534

5

15

153 
Listado de especies de diatomeas identificadas en un estudio taxonómico exhaustivo:

Achnanthidium microcephalum Kütz.

Achnanthidium minutissimum (Kütz.) Czrnecki

Achnanthidium saprophilum (Kobayasi \& Mayama) Round

\& Bukhtiyarova

Amphora copulata (Kütz.) Schoeman \& Arch.

Amphora inarensis Kramm.

Amphora ovalis (Kütz.) Kütz.

Amphora pediculus (Kütz.) Grun.

Amphora thumensis (mayer) Cl.-Euler

Amphora veneta var. capitata Haworth

Aneumastus stroessi (Oestr.) Mann \& Stickle

Anomoeoneis sphaerophora (Kütz.) Pfitz.

Asterionella formosa Hass.

Aulacoseira ambigua (Grun.) Sim.

Aulacoseira granulata (Ehr.) Sim.

Brachysira styriaca (Grun.) Ross

Brachysira vitrea (Grun.) Ross

Caloneis alpestris (Grun.) $\mathrm{Cl}$.

Cocconeis placentula Ehr. var. placentula

Cocconeis placentula var. euglypta (Ehr.) Grun.
Craticula halophila (Grun.) D. G. Mann

Cyclotella meneghiniana Kütz.

Cyclotella ocellata Pant.

Cyclotella aff. Praetermissa Lund

Cyclotella radiosa (Grun.) Lemm.

Cyclotella stelligera $\mathrm{Cl}$. \& Grun.

Cyclotella aff. wuethrichiana Druart \& Straub

Cyclostephanos invisitatus (Hohn \& Hellerman) Theriot,

Stoermer \& Hakansson

Cymbella affinis Kütz.

Cymbella cistula (Ehr.) Kirchner

Cymbella cymbiformis Ag.

Cymbella delicatula Kütz.

Cymbella helvetica Kütz.

Cymbella incerta (Grun.) Cl. Cymbopleura amphicephala

(Naeg.) Krammer

Cymbopleura hustedtii (Krasske) Krammer

Cymbopleura subaequalis (Grun.) Krammer

Denticula kuetzingii Brun

Encyonopsis aequalis

\section{RESULTADOS}

\section{Especies cosmopolitas y dominantes}

El estudio taxonómico del fitoplancton muestreado en las Lagunas de Ruidera desde mayo de 2000 hasta diciembre de 2001, mostró la presencia de 126 especies (Tabla 1) pertenecientes a los siguientes grandes grupos de algas (Reynolds, 1984): 9 taxones pertenecientes a Cyanophyta, 9 a Euglenophyta, 6 a Cryptophyceae, 7 a Dinophyceae, 10 a Chrysophyceae, 58 a Chlorophyta, 14 a Bacillariophyceae, 12 a Desmidiaceae, y un grupo de pequeños flagelados considerados como una única población debido a su pequeño tamaño y a la dificultad en su identificación. Un estudio taxonómico exhaustivo de las diatomeas reveló la existencia de 89 taxones más (Tabla 1), que o bien debido a su naturaleza bentónica o a su escasa presencia (indetectables por sedimentación), no fueron consideradas en los recuentos de fitoplancton.

En general, los taxones observados se consideran cosmopolitas en la bibliografía especializada. Las especies más comunes en las lagunas fueron: Cyclotella ocellata, que apareció en el 99\% de las muestras (total de muestras, total de meses), Rhodomonas minuta y Cryptomonas erosa en más del 90\%, Cyclotella kuetzingiana en el 85\% y Peridinium umbonatum en el 75\%. Otras poblaciones estuvieron presentes en más del $50 \%$, como Dinobryon sertularia, Cryptomonas marsonii y los pequeños flagelados. El $40 \%$ de los taxones fueron considerados esporádicos, ya que estuvieron presentes en menos del 5\% del total de muestras. De éstos, casi la mitad sólo aparecieron en una muestra. Los grupos taxonómicos con mayor número de especies poco presentes fueron las Euglenofitas, las Desmidiáceas y las Clorofíceas.

A pesar de no poder asegurar quienes fueron las especies dominantes en las lagunas debido a la baja frecuencia de muestreo, se puede destacar la importancia de algunas de ellas al suponer más del 70\% del biovolumen de fitoplancton en algunas muestras, como fue el caso de D. sertularia en Rey, Tetraselmis cordiformis en Morenilla y $C$. marsonii en Redondilla.

\section{Dinámicas de densidad y biovolumen del fitoplancton}

El rango de abundancia del fitoplancton en las Lagunas de Ruidera durante el período de estudio fue de $25 \cdot 10^{4}$ a $16 \cdot 10^{6} \mathrm{ind} / \mathrm{l}$, excepto en Tinaja, donde puntualmente se alcanzaron los $90 \cdot 10^{6} \mathrm{ind} / 1$. 
En general, no se encuentra una dinámica regular para el conjunto de las lagunas, pero sí se pudieron hacer una serie de agrupaciones de las mismas en función de un patrón temporal a lo largo del período de muestreos (Fig. 3):

En Colgada, Conceja y Tomilla se produjeron picos de densidad poco pronunciados en Septiembre 2000, debido a Synechocystis aqua-
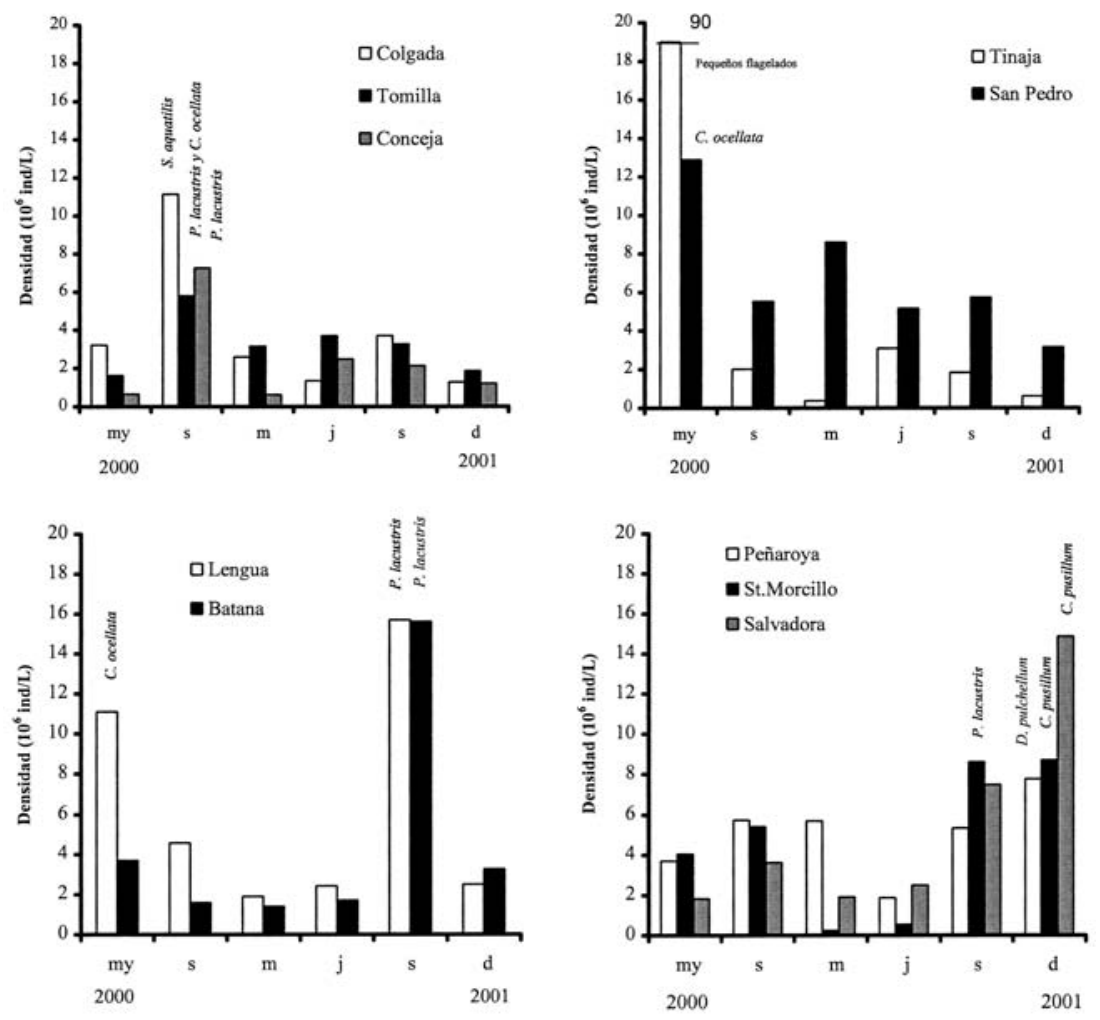

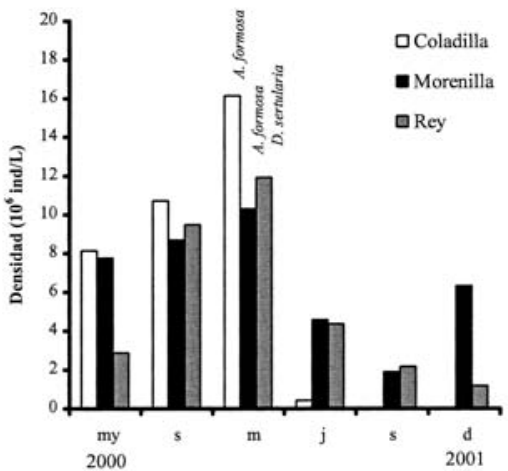

tilis y Planktonema lacustris. En Tinaja y San Pedro, se produjeron máximos en mayo: Tinaja destacó porque en ella se produjo el mayor pico de densidad de las lagunas, debido casi exclusivamente a pequeños flagelados; San Pedro presentó una densidad pronunciada en ambas primaveras, debido a $C$. ocellata y a los pequeños flagelados. Un tercer grupo (Lengua y Batana)

Figura 3. Densidad del fitoplancton total en cada laguna desde la primavera de 2000 al invierno de 2001. Se han agrupado las lagunas que presentan patrones temporales similares. Se indican las especies dominantes. Total phytoplankton density in each lagoon from spring 2000 to winter 2001. Lagoons with similar temporary patterns have been grouped. Dominant species are indicated. 
es el que presentó mayores abundancias en mayo y septiembre (distribución bimodal): en ambas se produjo el segundo valor máximo en el conjunto de las lagunas, en septiembre de 2001, debido a un florecimiento de P. lacustris. En un cuarto grupo se incluyen Peñarroya, Santo Morcillo y Salvadora, las únicas lagunas cuyo fitoplancton fue más abundante en diciembre. Las dos últimas ya presentaron valores altos en el septiembre anterior. Destacó Cosmarium pusillum. Un quinto grupo (Coladilla, Morenilla y Rey) presentó un aumento progresivo de la densidad de mayo de 2000 a marzo de 2001, descendiendo más acusadamente hasta diciembre. En este grupo destacó Asterionella formosa. Por último, Blanca, Redondilla y Cenagosa formarían, por su carácter temporal, otro grupo. En ellas no se superaron los $4 \cdot 10^{6}$ ind/l en ningún mes de estudio.

El biovolumen calculado del fitoplancton se encontró entre 0.2 y $7.2 \mathrm{~mm}^{3} / 1$. Un análisis de regresión reveló que la biomasa del fitoplancton mostró una correlación positiva y significativa $(\mathrm{p}<0.01)$ con la concentración de clorofila $a$, pero su relación con la concentración de fósforo total no fue significativa.

En el estudio de los promedios anuales de biovolumen del fitoplancton (Fig. 4), los máximos se dieron en Morenilla, San Pedro y Salvadora. Tinaja, Lengua y St. Morcillo también mostraron biovolumenes elevados. Los mínimos se produjeron en Blanca, Redondilla y Cenagosa. Con respecto a los promedios espaciales (Fig. 4), los máximos se produjeron en el 2000, siendo en el resto de meses inferior, y dándose el mínimo en marzo de 2001. Los análisis ANOVA de un factor revelaron que la media de la biomasa de primavera en las lagunas con poca cobertura de carófitos no fue significativamente distinta en aquellas con más cobertura.

Las variaciones temporales de los biovolúmenes se comentan siguiendo las agrupaciones anteriores. En Colgada, Tomilla y Conceja no se superaron los $2 \mathrm{~mm}^{3} / 1$, dándose el mayor biovolumen en verano. Sin embargo, Colgada presentó un máximo en Septiembre de 2000, con $4.5 \mathrm{~mm}^{3} / 1$, debido a $C$. erosa y $C$. ocellata.
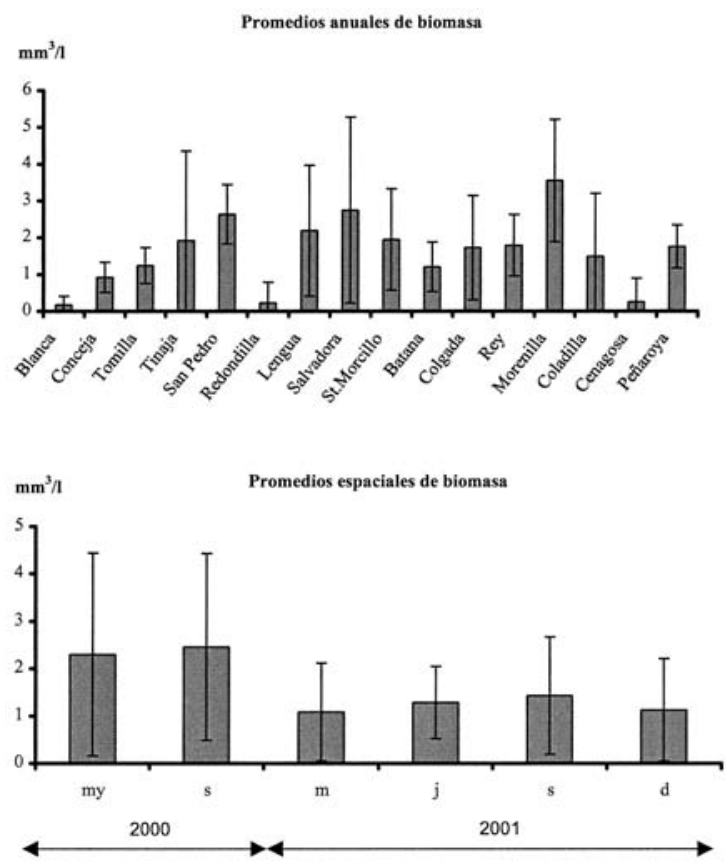

Figura 4. Promedios anuales y espaciales de la biomasa del fitoplancton en las lagunas de Ruidera durante el periodo 2000-2001. Las barras indican la amplitud de la desviación típica. Annual and spatial averages of phytoplankton biomass in Lagunas de Ruidera during period 2000-2001. Bars show the amplitude of typical deviation

En el grupo de Tinaja y San Pedro los máximos de biovolumen en primavera coincidieron con los de densidad. Tinaja presentó el segundo pico más importante de biovolumen de las lagunas: $7 \mathrm{~mm}^{3} / 1$, coincidiendo con el de densidad, debido precisamente a los pequeños flagelados, ya que estos supusieron casi el $70 \%$ del biovolumen. En el resto de meses no se alcanzaron los $2 \mathrm{~mm}^{3} / 1$, dándose mínimos en marzo y diciembre de 2001. San Pedro presentó un máximo de $4 \mathrm{~mm}^{3} / 1$ debido a $C$. ocellata. En los demás meses se superaron ampliamente los $2 \mathrm{~mm}^{3} / 1$, excepto en septiembre de 2001.

En Lengua y Batana, el biovolumen no sigue el patrón de distribución de la densidad. En Lengua, en mayo de 2000, C. ocellata y C. hirundinella representaron el 70\% del biovolumen total $\left(6 \mathrm{~mm}^{3} / 1\right)$. Sin embargo, su mayor densidad en el 2001 no se ve reflejada en un alto biovolumen, debido al pequeño tamaño de 
P. lacustris. Batana presentó bajos biovolúmenes todo el año, excepto septiembre de 2000 y junio de 2001, donde se alcanzaron máximos poco pronunciados $\left(2 \mathrm{~mm}^{3} / 1\right)$.

Peñarroya presentó un biovolumen entre $1 \mathrm{y}$ $2 \mathrm{~mm}^{3} / 1$ durante todo el año sin ningún patrón reconocible. Santo Morcillo se asemeja a Colgada, al presentar su fitoplancton un máximo de biovolumen en septiembre de 2000, de $4 \mathrm{~mm}^{3} / 1$, debido en un $60 \%$ a C. erosa. En Salvadora tuvo lugar el mayor biovolumen detectado en las lagunas, de $7 \mathrm{~mm}^{3} / 1$ en septiembre 2000 , que se debió a C. erosa y Peridinium willei en un $75 \%$. Coincidiendo con el aumento de densidad de septiembre a diciembre de 2001, el biovolumen también se incrementó, debido a la presencia de Cyclotella y Cosmarium en septiembre y a Cyclotella y Peridinium en diciembre.

Coladilla y Rey aumentaron su biovolumen en septiembre de 2000, siguiendo el patrón mostrado por su densidad; destacaron Cryptomonas phaseolus, P. umbonatum y A. formosa, cada una de
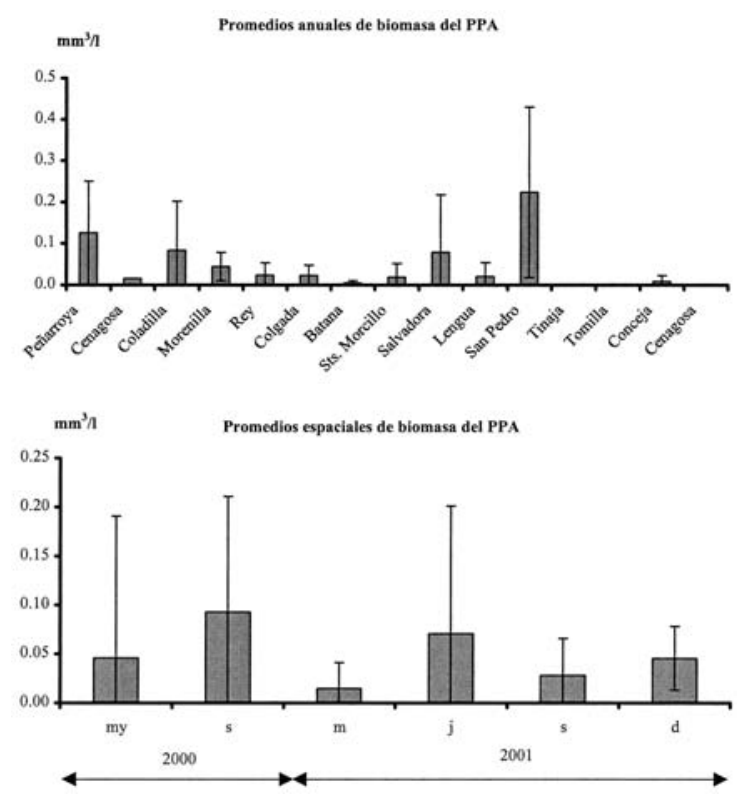

Figura 5. Promedios anuales y espaciales de la biomasa del picoplancton autotrófico en las lagunas de Ruidera durante el periodo 2000-2001. Las barras indican la amplitud de la desviación típica. Annual and spatial averages of autotrophic picoplankton biomass in Lagunas de Ruidera during the period 2000-2001. Bars show the amplitude of typical deviation. ellas con un $25 \%$ del biovolumen total $\left(4 \mathrm{~mm}^{3} / \mathrm{l}\right)$ en Coladilla, y C. erosa (50\%) en Rey. Esta última alcanzó el máximo en marzo $\left(3 \mathrm{~mm}^{3} / 1\right)$ debido al florecimiento de D. sertularia. Morenilla presentó una distribución temporal del biovolumen inversa a la de la densidad. P. willei causó su máximo $\left(6 \mathrm{~mm}^{3} / \mathrm{l}\right)$ en mayo de 2000 y un nuevo pico de biovolumen se dio en septiembre de 2001, debido a Tetraselmis cordiformis, una especie esporádica en las lagunas, ya que sólo apareció en otra muestra, Blanca (diciembre 2001).

Blanca presentó biovolúmenes inferiores a $0.5 \mathrm{~mm}^{3} / 1$; se observó una sustitución en la dominancia desde junio a diciembre de 2001: Oocystis sp. $\rightarrow$ P. umbonatum $\rightarrow$ C. erosa. En Redondilla se alcanzó $1.4 \mathrm{~mm}^{3} / 1$, debido casi exclusivamente a las criptofíceas C. marsonii (70\% del biovolumen), C. erosa y R. minuta. En Cenagosa, se alcanzó un biovolumen ligeramente inferior, debido a A. formosa y Synedra ulna, con un $35 \%$ cada una.

\section{Dinámicas de densidad y biovolumen del picoplancton autotrófico}

El rango de densidades del PPA fue desde $3.5 \cdot 10^{8} \mathrm{ind} / 1$ hasta niveles indetectables (en Tomilla y Tinaja). Las mayores densidades promedio se alcanzaron en San Pedro y Peñarroya $\left(3.5 \cdot 10^{8}\right.$ y $2.5 \cdot 10^{8} \mathrm{ind} / 1$, respectivamente), mientras en Coladilla, Morenilla y Salvadora se produjeron abundancias promedio próximas a $10^{8} \mathrm{ind} / \mathrm{l}$. El resto de lagunas presentaron abundancias menores, dándose los mínimos en Conceja y Batana ( $\left.10^{7} \mathrm{ind} / \mathrm{l}\right)$. Con lo que respecta a los promedios espaciales, las mayores densidades promedio se dieron en junio 2001 (1.4.10 ${ }^{8}$ ind/1). En el 2000 se alcanzaron densidades también elevadas, en torno a los $10^{8}$ ind/1. Los valores mínimos se dieron en marzo, con $3 \cdot 10^{7}$ ind/l.

Respecto a los promedios anuales de biovolumen (Fig. 5), los máximos se produjeron, al igual que en el caso de la densidad, en San Pedro y Peñarroya. En la primera los máximos de biovolumen se dieron en primavera del 2000 $\left(0.6 \mathrm{~mm}^{3} / 1\right)$ y en julio de $2001\left(0.4 \mathrm{~mm}^{3} / 1\right)$; en la segunda, también en junio con un biovolumen 
muy similar. En Salvadora, también se produjo un máximo muy parecido, pero en verano de 2000. Los valores mínimos sucedieron de nuevo en Conceja y Batana.

En contraste con los promedios espaciales de densidad, los biovolúmenes espaciales promedio máximos (Fig. 5) se dieron en septiembre de 2000, aunque en junio también fue elevado. El mínimo volvió a producirse en marzo 2001.

La contribución del picoplanton autotrófico a la biomasa del fitoplancton total fue en promedio del $2 \%$ (s.d. $4 \%$ ), con un mínimo de $0.001 \%$ y un máximo de $26 \%$ (siempre los máximos en Peñarroya y San Pedro).

\section{DISCUSIÓN}

Las concentraciones de fósforo total y de clorofila $a$ empleados para determinar el nivel trófico de las lagunas llevaron a la conclusión de que la mayoría de ellas son meso-eutróficas en función del primer indicador, y oligotróficas en función del segundo, según los criterios de eutrofia de la OCDE (1982). Esta diferencia en el grado de eutrofia entre ambos indicadores muestra el desfase existente entre nutrientes y biovolumen fitoplanctónico en Ruidera, encontrándose menos biomasa de la esperada para esos nutrientes, hecho que explica también la alta transparencia que existe en la columna de agua (Álvarez-Cobelas et al., 2002). Este aparente desfase y la falta de crecimiento del biovolumen planctónico en junio sugirió la siguiente hipótesis ya mencionada por Álvarez-Cobelas et al. (2002): la existencia de carófitos en las lagunas provoca una inhibición en el crecimiento algal fitopanctónico (Wium-Andersen et al., 1982). Sin embargo, con respecto a la biomasa del fitoplancton, y con los datos hasta ahora disponibles, el posible efecto alelopático de los carófitos no pudo ser demostrado.

Por otra parte, el hecho de que algunas lagunas reciban aguas contaminadas (aguas residuales domésticas y agrícolas de Ruidera y de Ossa de Montiel) influya sobre el nivel trófico de las mismas (Álvarez-Cobelas et al., 2002), no pare- ce ser la única explicación de los incrementos de biomasa del fitoplancton, ya que éstos ocurren en lagunas alejadas de los vertidos; es de suponer que la contaminación difusa (desde el acuífero) también esté interviniendo. Estos factores explicarían la gran heterogeneidad espacial del biovolumen. Con lo que respecta a la heterogeneidad temporal que presenta cada laguna, es propia de sistemas eutróficos (Marshall \& Petres, 1989), y coincide con la observada con la clorofila $a$.

El fitoplancton identificado en las lagunas es en general característico de otros lagos cársticos de la Península Ibérica (Dasí \& Miracle, 1991). Las especies identificadas en 1972 (Armengol et al., 1975) en las lagunas Colgada, Rey, Morenilla y Redondilla siguen apareciendo actualmente (o son morfotipos similares), aunque las características del presente estudio permitan establecer una mayor riqueza específica para las lagunas. Comparando el fitoplancton encontrado en las lagunas de Ruidera con otros sistemas cársticos próximos (Ciudad Real) como la laguna de La Cruz, de epilimniom mesotrófico (Dasí \& Miracle, 1991, Rodrigo et al., 2001), se observa una riqueza similar, pero una abundancia del orden de 10 veces superior a los máximos que se produjeron en La Cruz (Miracle et al., 1992).

Las asociaciones planctónicas (definidas por las especies dominantes) que pueden describir las lagunas fueron los siguientes grupos funcionales (según Reynolds, 1997): Y (Cryptomonas; pequeños lagos eutróficos), L (Peridinium umbonatum y Ceratium; lagos meso-eutróficos estratificados), C (Asterionella formosa y Aulacoseira; lagos eutróficos mezclados) y E (Dinobryon; lagos oligo-mesotróficos).

La contribución del picoplancton autotrófico a la biomasa total de fitoplancton resultó menor de lo esperado en sistemas meso-eutróficos (Bell \& Kalff, 2001); además, los valores de mayor contribución se dan en los lugares más eutróficos como el embalse de Peñarroya y la laguna San Pedro, tendencia inversa a la que algunos autores defienden (Vörös et al., 1998).

Las lagunas de Ruidera presentan una alta riqueza específica fitoplanctónica favorecida por 
las dinámicas de aislamiento y conexión entre ellas que ocurren a lo largo de los años. Esta riqueza es propia de ambientes aun no muy contaminados, ya que un endurecimiento de las condiciones propicia la dominancia de unas pocas tolerantes y disminuye la diversidad (Reynolds, 1997, Dodson et al., 2000). Sin embargo, la composición algal indica un proceso de eutrofización, aunque la biomasa de productor primario planctónico no sea todavía elevada. Un aumento de la biomasa fitoplanctónica, como respuesta a las concentraciones de nutrientes que ya tienen las lagunas, podría impedir el crecimiento de los macrófitos sumergidos (Scheffer et al., 1993) y llevar a las lagunas a un estado hipertrófico no deseable.

\section{AGRADECIMIENTOS}

Este trabajo se ha llevado a cabo gracias a la financiación del proyecto FEDER: 1-FD97-1812. Agradecemos la inestimable ayuda de las personas que trabajan en el Parque, desde la Dirección hasta la Guardería. Deseamos destacar la labor de campo y laboratorio de P. Riolobos, M. Álvarez Cobelas (CSIC) y la ayuda prestada por A. Comas (Academia de las Ciencias, Cuba) en la labor de identificación taxonómica de las clorofíceas. Así mismo agradecemos la ayuda de M. Lionard en la confección de este manuscrito.

\section{BIBLIOGRAFÍA}

ÁLVAREZ-COBELAS, M. 2003. La calidad de las aguas de baño en las lagunas de Ruidera. Revista de Estudios Albacetenses, 4: 173-222. Albacete.

ÁlVAREZ-COBELAS, M., R. CASTELLÓ, S. CIRUJANO, C. ROJO \& M. A. RODRIGO. 2002. La contaminación de las lagunas de Ruidera. Actas II Simposio del Medio Natural Albacetense (en prensa), Albacete.

ANDREWS, J. E., M. PEDLEY \& P. F. DENNIS. 2000. Palaeoenvironmental records in Holocene Spanish tufas: a stable isotope approach in search of reliable climatic archives. Sedimentology, 47 (7): 961-978.

ANTON, A. \& H. DUTHIE. 1981. Use of cluster analisys in the systematics of the algal genus Cryptomonas. Can. J. Bot., 59: 992-1002.
ARAGÓN, J. R. 2001. La protección normativa de los humedales de la cuenca del Alto Guadiana. En: Lagunas Manchegas. J. R. Aragón, S. Cirujano, M. Velasco \& A. Trujeque (eds.): 11-70. Aguas de Alcázar, E.M.S.A., Alcázar de San Juan, España.

ARMENGOL, X. \& M. R. MIRACLE. 2000. Diel vertical movements of zooplankton in lake la Cruz (Cuenca, Spain). J. Plankton Res., 22: 1683-1703

ARMENGOL, J., M. ESTRADA, A. GUISET, R. MARGALEF, D. PLANAS, J. TOJA \& F. VALLESPINÓS. 1975. Observaciones limnológicas en las lagunas de La Mancha. Bol. Est. Central. Ecol., 4 (8): 11-27.

BELL, T. \& J. KALFF, 2001. The contribution of picophytoplankton in marine and freshwater systems of different trophic status and depth. Limnol. Oceanogr., 46: 1243-1248.

COMAS, A. 1996. Las Chlorococcales dulciacuicolas de Cuba. J. Cramer, Stuttgart.

COMAS, A. \& L. KRIENITZ. 1997. Comparative LMand SEM- studies on Coelastrum (Chlorophyta, Chlorellales) under culture conditions. Algological studies, 87:87-98.

DASÍ, M. J. \& M. R. MIRACLE. 1991. Distribución vertical y variación estacional del fitoplancton de una laguna cárstica meromíctica, la laguna de la Cruz (Cuenca, España). Limnetica, 7: 37-59.

DODSON, S. I., S. E. ARNOTT \& K. L. COTTINGHAM. 2000. The relationship in lake communities between primary productivity and species richness. Ecology, 81: 2662-2679.

ELVIRA, B., G. G. NICOLA \& A. ALMODOVAR. 1996. Pike and red swamp crayfish: A new case on predator-prey relationship between aliens in central Spain. J. Fish Biol., 48 (3): 437-446.

GARCÍA, M. A., J. A. GONZÁLEZ \& S. ORDÓÑEZ. 1997. Geología y Geomorfología. En: Parque Natural Lagunas de Ruidera. Vicente García Canseco (ed.): 20-50. Ecohábitat, España.

GRANDE, F. 1997. Hidrogeología. En: Parque Natural Lagunas de Ruidera. Vicente García Canseco (ed.): 52-68. Ecohábitat, España.

GRANDE, F. 1997. Hidrogeología. En: Parque Natural Lagunas de Ruidera. Vicente García Canseco (ed.): 52-68. Ecohábitat, España.

HABDIJA, I. 1997. Longitudinal distribution of predatory benthic macroinvertebrates in a karstic river. Arch. Hydrobiol., 139 (4): 527-546.

HASLE, G. R. \& FRYXELL, G. A. 1970. Diatoms: cleaning and mounting for light and electron microscopy. Trans. Am. Microscop. Soc., 89 (4): 469-474.

HINDÁK, F. 1987. Taxonomic survey of the genera Fusola (Chlorococcales), Elakatothrix, Closteriospira 
and Chadefaudiothrix (Ulotrichales). Preslia, Praha, 59: 193-228.

KOMÁREK, J. \& V. JANKOVSKÁ. 2001. Rewiev of the Green Algal Genus Pediastrum; Implication for Pollen analytical Research. Bibliotheca Phycol., 108. 127 pp.

LUND, J. W. G., C. KIPLING \& E. D. LE CREEN. 1958. The inverted method of estimating algal numbers and the statistical basis of estimation by counting. Hydrobiol., 11: 143-170.

MACISAAC, A. \& J. G. STOCKNER. 1993. Enumeration of Phototrophic Picoplankton by Autofluorescence Microscopy. In: Handbook of Methods in Aquatic Microbial Ecology: 187-197. Lewis Publishers. Boca Raton (FL), USA.

MARSHALL, C. T. \& R. H. PETERS. 1989. General patterns in the seasonal development of chlorophyll $a$ for temperate lakes. Limnol. Oceanogr., 34 (5): 856-867.

MATONICKIN, R., I. HABDIJA \& B. PRIMC-HABDIJA. 2001. The effects of season and food availability on macroinvertebrate colonization in a woodland stream. Arch. Hydrobiol., 153 (1): 55-74.

MIRACLE, M. R., E. VICENTE \& C. PEDRÓS-ALIÓ. 1992. Biological studies of Spanish meromictic and stratified karstic lakes. Limnetica, 8: 59-77.

MONTERO, E. 2000. Contribución al estudio de la geometría y los límites del acuífero del Campo de Montiel. Instituto de Estudios Albacetenses "Don Juan Manuel". 177 pp.

OCDE. 1982. Eutrophication of Waters: monitoring, assessment and control. OCDE, París.

PEDLEY, M., J. ANDREWS, S. ORDONEZ, M. A. G. DEL CURA, J. A. G. MARTIN \& D. TAYLOR. 1996. Does climate control the morfological fabric of freshwater carbonates? A comparative study of Holocene barrage tufas from Spain and Britain. Palaeogeography-Palaeoclimatology-Palaeoecology, 121 (34): 239-257.

PERDICES, A., I. DOADRIO, I. M. COTE, A. MACHORDOM, P. ECONOMIDIS \& J. D. REYNOLDS. 2000. Genetic divergence and origin of Mediterranean populations of the River Blenny Salaria fluviatilis (Teleostei: Blenniidae). Copeia, 4 (3): 723-731.

PRIMC-HABDIJA, B., I. HABDIJA, M. MESTROV \& I. RADANOVIC. 1996. Composition of ciliate fauna and its seasonal changes in fluvial drift. Aquat. Sci., 58 (3): 224-240.

PRIMC-HABDIJA, B., I. HABDIJA, A. PLENKOVICMORAJ. 2001. Tufa deposition and periphyton overgrowth as factors affecting the ciliate community on travertine barriers in different current velocity condi- tions. Hydrobiologia, 457: 87-96.

REYNOLDS, C. S. 1984. The ecology of Freshwater Phytoplankton. Cambridge University Press, Cambridge, UK. 384 pp.

REYNOLDS, C. S. 1997. Vegetation processes in the pelagic: a model for ecosystem theory. Ecology Institute, Oldendorf-Luhe,Germany. $371 \mathrm{pp}$.

RIOLOBOS, P., M. ÁlVAREZ COBELAS, C. ROJO, M. A. RODRIGO, E. ORTEGA MAYAGOITIA \& S. CIRUJANO. 2002. Técnicas habituales de análisis fisicos, químicos y biológicos del Grupo de Investigación del Agua. Real Jardín Botánico CSIC, España.

RODRIGO, M. A., E. VICENTE \& M. R. MIRACLE. 2000. The role of light and concentration gradients in the vertical stratification and seasonal development of phototrophic bacteria in a meromictic lake. Arch. Hydrobiol. 148 (4): 533-548.

RODRIGO, M. A., M. R. MIRACLE \& E. VICENTE. 2001. The meromictic Lake La Cruz (Central Spain). Patterns of stratification. Aquat. Sci., 63: 406-416.

ROSALUZ, E. N. \& A. COMAS. 2000. Chlorococcalean algae (s. l.) from the Ecological Park of Xochimilco, Mexico. Algological Studies, 100:65-94.

ROJO, C. \& M. R. MIRACLE. 1987. Poblaciones fitoplanctónicas de la laguna de la Cruz (Cuenca), una laguna cárstica meromíctica. Actas del VI Simposio Nacional de Botánica Criptogámica, Granada, España: 119-137.

ROTT, E. 1981. Results from phytoplankton counting intercalations. Botanischer Institut, Universität Innsbruck., Austria.

SERRANO, M. A. \& J. L. GARCÍA. 1997. Clima. En: Parque Natural Lagunas de Ruidera. Vicente García Canseco (ed.): 70-88. Ecohábitat, España.

SCHEFFER, M., S. H. HOSPER, M. L. MEIJER, B. MOSS, E. JEPPESEN. 1993. Alternative equilibria in shallow lakes. Tree., 8:275-279.

SOKAL, R. R. \& F. J. ROHLF. 1986. Introducción a la bioestadística. Reverté, Barcelona. 362 pp.

TELL, G. \& V. GARCÍA. 1986. Euglenophyta Pigmentadas de la Argentina. J. Cramer. Buenos Aires, Argentina. 300 pp.

VÖRÖS, I., C. CALLIERI, K. V. BALOGH \& R. BERTONI, 1998. Freshwater picocyanobacteria along a trophic gradientr and light quality. Hydrobiologia, 369/370: 117-125

WIUM-ANDERSEN, S., U. ANTHONI, C. CHRISTOPHERSEN \& G. HOUEN. 1982. Allelopathic effects on phytoplankton by substances isolated from aquatic macrophytes (Charales). Oikos, 39:187-190. 
